CLINICAL STUDY

\title{
Hyperthyroidism and suicide: a retrospective cohort study in Sweden
}

\author{
Mirna Abraham-Nordling ${ }^{1,6}$, Stefan Lönn ${ }^{2}$, Göran Wallin ${ }^{1}$, Li Yin ${ }^{2}$, Olof Nyren ${ }^{2}$, Owe Tullgren ${ }^{3}$, Per Hall ${ }^{2}$ \\ and Ove Törring ${ }^{4,5}$ \\ ${ }^{1}$ Department of Molecular Medicine and Surgery. Karolinska Institutet, 17176 Stockholm, Sweden, ${ }^{2}$ Department of Medical Epidemiology and \\ Biostatistics, Karolinska Institutet, Stockholm, Sweden, ${ }^{3}$ Department of Oncology, Karolinska University Hospital, Solna, Sweden, ${ }^{4}$ Department of Clinical \\ Research and Education Sodersjukhuset, Karolinska Institutet, Stockholm, Sweden, ${ }^{5}$ Division of Endocrinology, Department of Internal Medicine, \\ Sodersjukhuset, Stockholm, Sweden and ${ }^{6}$ Division of Surgery, Department of Clinical Sciences, Karolinska Institutet, Danderyd Hospital, Stockholm, \\ Sweden \\ (Correspondence should be addressed to M Abraham-Nordling; Email: mirna.abraham.nordling@ki.se)
}

\begin{abstract}
Objectives: Mental symptoms and impaired quality of life commonly occur in patients treated for hyperthyroidism. Our aim was to determine whether a history of hyperthyroidism implies an increased risk of suicide.

Design: Historic cohort study of 43633 patients treated with radioiodine or surgery for hyperthyroidism between 1950 and 2005. The majority of the radioiodine-treated patients came from Stockholm. Two comparison cohorts consisted of 44921 patients registered with an operation for atoxic nodular goitre between 1965 and 2005, and 354861 patients with a cholecystectomy between 1965 and 2001.

Methods: Subjects were followed from the date of diagnosis until death, emigration or end of follow-up. Information on outcome was obtained from population and health registers.

Results: The number of observed suicide deaths was 134 in the study cohort. A naive comparison with the age-, sex- and calendar period-matched general Swedish population yielded a moderately increased standardized mortality ratio (SMR), but stratifications revealed that the excess was mainly driven by women from Stockholm, whose baseline suicide risk was higher than for women in the rest of Sweden. Using the population rates from Stockholm, the SMR among Stockholm women with Graves' disease was 1.14 (95\% confidence intervals (CI) $0.66-1.86)$ and toxic goitre 0.99 (95\% CI 0.51-1.72). A direct comparison between the study cohort and the combined comparison cohorts, with multiple adjustments (including adjustment for residence in Stockholm), yielded a relative risk of suicide of 0.93 (0.68-1.26).

Conclusions: This study did not confirm an increased risk of suicide among patients treated for hyperthyroidism.
\end{abstract}

European Journal of Endocrinology $160437-441$

\section{Introduction}

Hyperthyroidism is caused by excessive TH concentrations due to increased synthesis and secretion from the thyroid. It is a common disease and affects $\sim 2 \%$ of women and $0.2 \%$ of men (1). A recent survey has shown an annual incidence of 32.7/100 000 adults in Stockholm county (2). Mental symptoms such as nervousness, anxiety, poor concentration and personality changes are prominent (3-7). Long-term neuropsychiatric symptoms following hyperthyroidism have been shown even in patients who have been successfully treated (8-10). Several studies have also shown that the quality of life in many patients is severely impaired at long-term followup (10-12). Patients with previous Graves' disease in particular seem affected, since 32-56\% still felt tired, 23$38 \%$ gloomy and sad, $15-32 \%$ reported that the disease still affected them socially and 16-37\% felt that they were 'worn-out' even 14-21 years after treatment (12). Depressed patients seem to have altered TSH response to TRH, which has been suggested to be associated with the risk of suicide (13). In addition, THs may also interact with important mood modulating neurotransmitter systems like the serotonin system $(14,15)$.

In a previous study of Graves' disease (12), we observed that 7 patients out of 179 had died, 2 of whom had committed suicide. In the present study, we aimed to test the hypothesis that hyperthyroidism increases the risk of suicide using a nationwide population-based cohort. 


\section{Methods}

The study cohort included 44234 individuals diagnosed with hyperthyroidism between 1950 and 2005 in Sweden. It consisted of two approximately equally sized subcohorts - one with individuals who had been treated with ${ }^{131}$ I for hyperthyroidism in 1950-2000, of whom the majority were included after 1975 and consisted almost exclusively of patients from Stockholm (16) and one subcohort with patients identified in the Swedish inpatient registry as having undergone surgery for hyperthyroidism between 1965 and 2005. The two subcohorts included Graves' disease and toxic uni- or multinodular goitre, as well as patients who had toxic nodular goitre with Graves' disease. As a comparison cohort, we identified 45655 patients with a record of surgically treated atoxic goitre in the Swedish inpatient register between 1965 and 2005 (atoxic goitre cohort). As a second comparison cohort, we identified 354861 patients registered in the inpatient register as having undergone cholecystectomy between 1965 and 2001 (cholecystectomy cohort).

Using the national registration numbers (NRNs) assigned to all residents in Sweden shortly after birth or immigration as unique personal identifiers, we linked the cohorts to the registers of total population, migration and causes of death for correct censoring. We excluded 601734 and 16270 records in the study cohort, atoxic goitre cohort and cholecystectomy cohort respectively, due to erroneous NRNs or other inconsistencies revealed during the record linkages. Thus, the study cohort comprised 43633 patients, the atoxic goitre cohort 44921 patients and the cholecystectomy cohort 338591 patients (Table 1). All cohort members were followed from the date of first hyperthyroidism diagnosis (or 1 January 1961 for the earlier first diagnosis due to unreliable death registry data before that date), atoxic goitre diagnosis or cholecystectomy until death, emigration or end of study
(31 December 2005 for the study cohorts and the atoxic goitre cohort; 31 December 2001 for the cholecystectomy cohort), whichever occurred first. Underlying and contributory causes of death among deceased individuals were obtained from the essentially complete causes of death register, in turn based on obligatory death certificates issued by physicians.

In order to estimate the relative risk (RR) of suicidal death among hyperthyroidism patients in our study cohort, relative to the age-, gender- and calendar period-matched general Swedish population, we calculated the standardized mortality ratio (SMR), which is the ratio of observed to expected numbers of deaths due to suicide in the study cohort. The expected number was calculated by multiplying the mortality rates for suicide in the general Swedish population (divided into strata of 5-year age groups, gender and 5-year calendar periods) by the stratum-specific person-time accrued in the cohort. We calculated 95\% confidence intervals (CI) of the SMRs by assuming that the observed number of suicidal deaths followed a Poisson distribution. Stratified analyses were performed by gender, age at diagnosis, follow-up time from diagnosis, calendar period of followup and place of residence (Stockholm versus rest of Sweden). Since the hyperthyroidism is usually more severe, has a faster onset (month) and has an autoimmune background in Graves' disease compared with the clinically milder and slowly developing (years) toxic nodular goitre, separate analyses were performed for the two diseases. As it was suspected that an increased suicide rate could be an unspecific phenomenon linked to the hospitalization as such, we also did direct comparisons between the study cohort andthe comparison cohorts using Poisson regression, restricting to the follow-up period from 1965 to 2001 (for the cholecystectomy cohort, the end of follow-up was 2001) and adjusting for sex, age, time of inclusion into the cohorts, follow-up time, treatment modality (surgery or non-surgical treatment), recorded hospitalizations

Table 1 Overview of the three cohorts.

\begin{tabular}{llll}
\hline & All hyperthyroid & Atoxic goitre & Cholecystectomy \\
\hline Number of subjects & 43633 & 44921 & 338591 \\
Women & $36743(84 \%)$ & $37772(84 \%)$ & $227654(67 \%)$ \\
Men & $6890(16 \%)$ & $7149(16 \%)$ & $110937(33 \%)$ \\
Mean age (s.D.) & $51.3(16.8)$ & $50.5(14.3)$ & $52.8(16.6)$ \\
Diagnosis & $25391(58 \%)$ & & \\
Graves' disease & $16331(37 \%)$ & & \\
Toxic nodular goitre & $1911(4 \%)$ & & 55119 \\
Toxic nodular goitre with & & 5684 & 383472 \\
$\quad$ Graves' hyperthyroidism (mixed) & 14591 & 39237 & $1965-2001$ \\
Place of residence & 29042 & $1965-2005$ & 4647275.53 \\
Stockholmers & $1950-2005$ & 746390.25 & 1002 \\
Non-stockholmers & 703126.14 & 102 & \\
Years at cohort entry & 134 & & \\
Follow-up time (Person-years) & & \\
Number of suicides & &
\end{tabular}


for psychiatric diseases prior to inclusion into the cohort (albeit this information might have been incomplete due to left truncation) and place of residence (Stockholm versus rest of the country).

The study was approved by the local ethics committee of Karolinska Institutet.

\section{Results}

Mean age at entry was $51.3,50.5$ and 52.8 years in the study cohort, atoxic goitre and cholecystectomy comparison cohorts respectively (Table 1). Women were in the majority in all cohorts ( $84 \%$ in both thyroid cohorts and $67.2 \%$ in the cholecystectomy cohort). A diagnosis of Graves' disease had been given to 25391 patients, 16331 were classified as having toxic nodular goitre and 1911 were recorded with both toxic nodular goitre and Graves' disease. A total of 134 (30 male and 104 female) suicide deaths were identified in the study cohort versus 108 expected, corresponding to an overall SMR of 1.24 (95\% CI 1.04-1.47; Fig. 1). The excess mortality due to suicide was confined to women $(\mathrm{SMR}=$ $1.41,95 \%$ CI 1.16-1.71), whereas the point estimate of SMR among men was 0.86 (95\% CI 0.58-1.23). Stratification into Graves' disease versus toxic nodular goitre revealed that the excess was essentially confined to Graves' disease (SMR 1.35, 95\% CI 1.07-1.66), while the SMR for toxic nodular goitre was 1.06 (95\% CI 0.77-1.43).

Since the suicide rates for women in Stockholm County differ from those for women in the rest of Sweden, and Stockholmers were overrepresented in our study cohort, we stratified the female part of the cohort into residents of Stockholm and residents of the rest of Sweden. Naively using the nationwide Swedish population rates, Stockholm women with either main type of hyperthyroidism seemingly had substantial excesses of suicide (point estimates of SMRs associated with total hyperthyroidism, Graves' disease and toxic nodular goitre were $1.60,1.56$ and 1.47 respectively). We therefore recalculated SMRs for Stockholm and nonStockholm women using suicide rates specific for Stockholm County and for all of Sweden respectively. This analysis had to be restricted to women who entered the cohort in 1975 or later due to unavailability of specific Stockholm rates before this year. Figure 1 reveals that the excess among Stockholm women in the study cohort virtually disappeared when we used the appropriate Stockholm population rates. The SMRs for Stockholm women with any hyperthyroidism, Graves' disease and toxic nodular goitre were 1.10 (95\% CI 0.73-1.58), 1.14 (95\% CI 0.66-1.86) and 0.99 (95\% CI 0.51-1.72) respectively (Table 2).

Also non-Stockholm women with Graves' disease appeared to exhibit a sizeable excess when we analysed SMR using the national population rates. However, these women represented the oldest part of the radioiodine cohort, with hyperthyroidism diagnoses dating as far back as 1950. As this old cohort was not evenly distributed across non-Stockholm Sweden, we could not exclude bias through geographic mismatch between the observed suicide rate and the population rate (all of Sweden) used for comparison. Moreover, the validity of suicide diagnoses in the death register during the 1960 s and early 1970s is less well documented. We therefore made a restriction to non-Stockholm women with Graves' disease who entered the cohort in 1975-2005. They were all identified in the Swedish inpatient register, which attained $75 \%$ coverage of the Swedish population in 1978. Then the SMR was 1.38 (95\% CI 0.80-2.20).

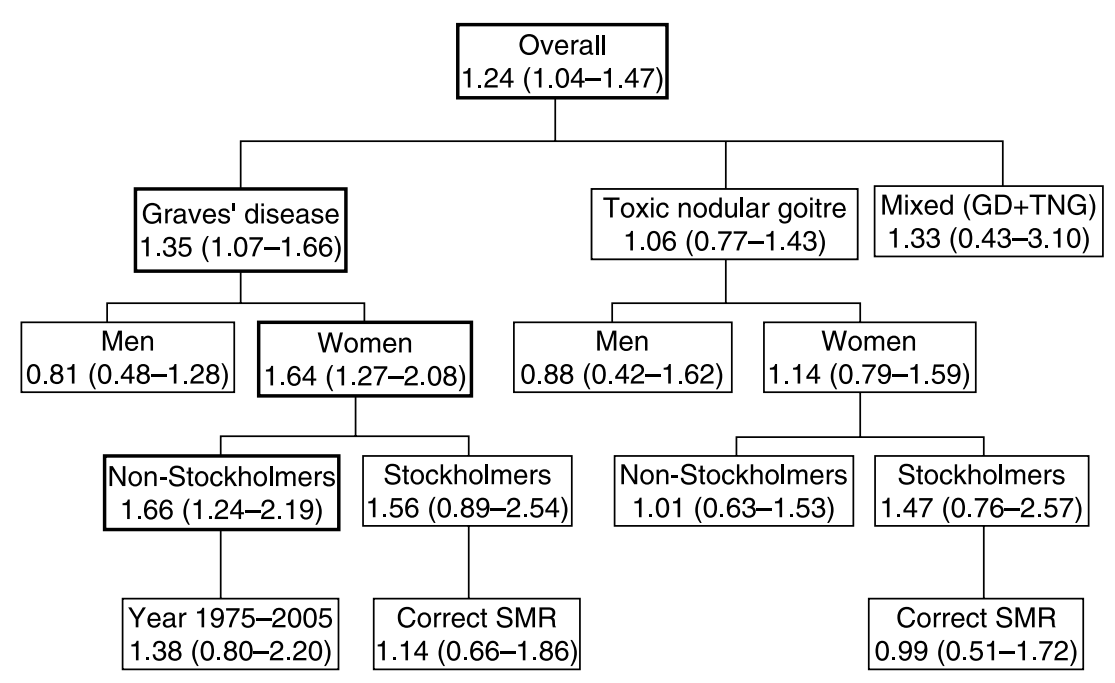

Figure 1 Stratification of the standardized mortality ratios (SMR) and $95 \%$ confidence intervals $(\mathrm{Cl})$ of the study cohort. 
Table 2 Poisson regression model to estimate the relative risk (RR) of suicide with confidence intervals $(95 \% \mathrm{Cl})$.

\begin{tabular}{|c|c|}
\hline & $\mathbf{R R}(95 \% \mathrm{Cl})$ \\
\hline \multicolumn{2}{|l|}{ Disease $^{a}$} \\
\hline Hyperthyroidism & $0.93(0.68-1.26)$ \\
\hline Cholecystectomy or atoxic nodular goitre & 1.00 (Reference) \\
\hline \multicolumn{2}{|l|}{ Follow-up time } \\
\hline 1 year & $1.38(1.10-1.73)$ \\
\hline $1-4$ year & $1.08(0.92-1.26)$ \\
\hline $5-9$ year & $1.09(0.93-1.27)$ \\
\hline $10+$ year & 1.00 (Reference) \\
\hline \multicolumn{2}{|l|}{ Attained age } \\
\hline$>75$ & $1.32(1.08-1.60)$ \\
\hline $65-74$ & $0.97(0.80-1.18)$ \\
\hline $55-64$ & $0.97(0.80-1.17)$ \\
\hline $45-54$ & $1.13(0.94-1.37)$ \\
\hline $00-44$ & 1.00 (Reference) \\
\hline \multicolumn{2}{|l|}{ Sex } \\
\hline Men & $2.73(2.43-3.08)$ \\
\hline Women & 1.00 (Reference) \\
\hline \multicolumn{2}{|l|}{ Residence area } \\
\hline Other area & $0.90(0.77-1.05)$ \\
\hline Stockholm county & 1.00 (Reference) \\
\hline \multicolumn{2}{|l|}{$\begin{array}{l}\text { Year of entry into cohorts } \\
\text { (year of diagnosis or cholecystectomy) }\end{array}$} \\
\hline Before 1979 & $1.79(1.47-2.18)$ \\
\hline 1980-1989 & $1.30(1.06-1.59)$ \\
\hline $1990-$ & 1.00 (Reference) \\
\hline \multicolumn{2}{|l|}{ Treatment mode } \\
\hline Non-surgery & $1.19(0.72-1.99)$ \\
\hline Surgery & 1.00 (Reference) \\
\hline \multicolumn{2}{|l|}{ Psychiatric disease before entry to cohort } \\
\hline Yes & $7.39(6.31-8.64)$ \\
\hline No & 1.00 (Reference) \\
\hline
\end{tabular}

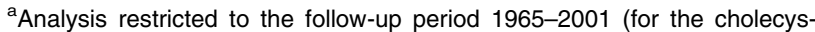
tectomy cohort, the end of follow-up was 2001) and with mutual adjustments for all variables in the table.

In direct comparison between the study cohort and the combined comparison cohorts using Poisson regression with multiple adjustments, the RR among the former with the latter as reference was 0.93 (0.68-1.26).

\section{Discussion}

Among patients who had been treated with ${ }^{131} \mathrm{I}$ or surgery for hyperthyroidism in Sweden from 1950 to 2005 , we observed a moderately increased risk of death due to suicide, when naively using population rates from the entire Swedish population. Because the suicide rate in the general female population of Stockholm has been $\sim 50 \%$ higher than the corresponding rate in the entire Swedish female population during the study period (17), the excess among Stockholm women essentially disappeared (RR for Graves' disease and toxic nodular goitre was 1.14 and 0.99 respectively) when we used the appropriated background rates. A direct comparison with hospitalized comparison cohorts (thyroidectomy for atoxic nodular goitre and cholecystectomy), which should have removed much of the possible unspecific effects of the hospitalization or the treatment per se adjusting for a number of possible confounding factors including hospitalizations for psychiatric disease prior to inclusion, yielded a RR of 0.93 .

Given the absence of any important elevations of suicide mortality in any other stratum (men, Stockholm women and toxic nodular goitre), the unexplained excess observed among non-Stockholm women with Graves' disease appears as an outlier result. Clearly, geographic mismatch between our cohort and the nationwide population rates explained some of this excess, which fell from 1.66 to 1.38 (non-significant) when we restricted our analysis to women included after 1974 (and thus improved - albeit not perfected the geographic matching). Although we cannot exclude a true association, we interpret the remaining nonsignificant $38 \%$ excess as a chance finding.

Strengths of our study include the cohort design with practically all patients who received ${ }^{131} \mathrm{I}$ treatment for hyperthyroidism in large parts of Sweden after 1950 and essentially all patients who were surgically treated for hyperthyroidism in Sweden after the mid 1980s.

Limitations to be highlighted include the absence of information about possible confounding factors, notably psychiatric status prior to diagnosis. Cross-linkage within the inpatient register made it possible for us to identify hospitalizations for psychiatric disease among some of our cohort members (at least after 1973 when the mental hospitals were connected to the registration), but due to left truncation and the unavailability of information about psychiatric outpatient care gives scope for residual confounding. However, negative confounding that has cancelled a true association seems unlikely. It should be noted that we did not adjust for psychiatric disease after onset of hyperthyroidism, because it could be in the causal chain to suicide. Moreover, we did not count suicide attempts because of the notorious difficulties with misclassification (17). If the case fatality among patients with suicide attempts would differ between our study and comparison cohorts, the comparison of suicide deaths may be a poor indicator of differences in the propensity to commit suicide. It should also be kept in mind that there is misclassification of fatal suicides, and a nonnegligible proportion is classified as accidental deaths. Furthermore, although the number of patients in the cohort was large, the number of observed events was less impressive, at least in substrata, and our ability to detect small but clinically important excess risks was somewhat limited.

In conclusion, this study did not confirm our prior hypothesis of an increased risk suicide among patients with treated hyperthyroidism.

\section{Declaration of interest}

There is no conflict of interest that could be perceived as prejudicing the impartiality of the research reported. 


\section{Funding}

The study was supported by the Swedish Research Council and Karolinska Institutet.

\section{Acknowledgements}

The authors would like to thank Curt Lennart Spetz at National Board of Health and Welfare, for performing pilot explorations in the Swedish Inpatient Register and Dr Guo-Xin Jiang, NASP, Karolinska Institutet for helping us with the population rates specific for Stockholm County.

\section{References}

1 Tunbridge WM, Evered DC, Hall R, Appleton D, Brewis M, Clark F, Evans JG, Young E, Bird T \& Smith PA. The spectrum of thyroid disease in a community: the Whickham survey. Clinical Endocrinology 19777 481-493.

2 Abraham-Nordling $\mathrm{M}$, Torring $\mathrm{O}$, Lantz $\mathrm{M}$, Hallengren $\mathrm{B}$, Ohrling H, Lundell G, Calissendorff J, Jorneskog G \& Wallin G. Incidence of hyperthyroidism in Stockholm, Sweden, 2003-2005. European Journal of Endocrinology 2008158 823-827.

3 Lazarus JH. Hyperthyroidism. Lancet 1997349 339-343.

4 Cooper DS. Hyperthyroidism. Lancet 2003362 459-468.

5 Weetman AP. Graves' disease. New England Journal of Medicine 2000343 1236-1248.

6 Placidi GP, Boldrini M, Patronelli A, Fiore E, Chiovato L, Perugi G \& Marazziti D. Prevalence of psychiatric disorders in thyroid diseased patients. Neuropsychobiology 199838 222-225.

7 Torring O, Tallstedt L, Wallin G, Lundell G, Ljunggren JG, Taube A, Saaf M \& Hamberger B. Graves' hyperthyroidism: treatment with antithyroid drugs, surgery, or radioiodine - a prospective, randomized study. Thyroid Study Group. Journal of Clinical Endocrinology and Metabolism 199681 2986-2993.
8 Stern RA, Robinson B, Thorner AR, Arruda JE, Prohaska ML \& Prange AJ Jr. A survey study of neuropsychiatric complaints in patients with Graves' disease. Journal of Neuropsychiatry and Clinical Neurosciences 19968 181-185.

9 Perrild H, Hansen JM, Arnung K, Olsen PZ \& Danielsen U. Intellectual impairment after hyperthyroidism. Acta Endocrinologica 1986112 185-191.

10 Fahrenfort JJ, Wilterdink AM \& van der Veen EA. Long-term residual complaints and psychosocial sequelae after remission of hyperthyroidism. Psychoneuroendocrinology 200025 201-211.

11 Ljunggren JG, Torring O, Wallin G, Taube A, Tallstedt L, Hamberger B \& Lundell G. Quality of life aspects and costs in treatment of Graves' hyperthyroidism with antithyroid drugs, surgery, or radioiodine: results from a prospective, randomized study. Thyroid $1998 \mathbf{8} 653-659$.

12 Abraham-Nordling M, Torring O, Hamberger B, Lundell G, Tallstedt L, Calissendorff J \& Wallin G. Graves' disease: a longterm quality-of-life follow up of patients randomized to treatment with antithyroid drugs, radioiodine, or surgery. Thyroid 200515 1279-1286.

13 Musselman DL \& Nemeroff CB. Depression and endocrine disorders: focus on the thyroid and adrenal system. British Journal of Psychiatry $199630123-128$.

14 Bauer M, Heinz A \& Whybrow PC. Thyroid hormones, serotonin and mood: of synergy and significance in the adult brain. Molecular Psychiatry 20027 140-156.

15 Kirkegaard C \& Faber J. The role of thyroid hormones in depression. European Journal of Endocrinology 1998138 1-9.

16 Holm LE, Hall P, Wiklund K, Lundell G, Berg G, Bjelkengren G, Cederquist E, Ericsson UB, Hallquist A, Larsson LG, Lidberg M, Lindberg S, Tennvall J, Wicklund H \& Boice JD. Cancer risk after iodine-131 therapy for hyperthyroidism. Journal of the National Cancer Institute 199183 1072-1077.

$17 \mathrm{http}: / / \mathrm{ki} . \mathrm{se} / \mathrm{ki} / \mathrm{jsp} /$ polopoly.jsp?d=18803\&l=sv

Received 11 December 2008

Accepted 30 December 2008 